\title{
28 Research Soure \\ Dynamics of SARS-CoV-2 transmission among evacuees quarantined at Jaisalmer, India
}

\section{Suman Saurabh ( $\nabla$ drsumansaurabh@gmail.com )}

World Health Organization - India https://orcid.org/0000-0003-2281-6783

\section{Ritesh Kumar}

CSIR Central Scientific Instruments Organisation

\section{Nitesh Kumar}

All India Institute of Medical Sciences - Jodhpur

\section{Pankaj Bhardwaj}

All India Institute of Medical Sciences - Jodhpur

\section{Vijaya Lakshmi Nag}

All India Institute of Medical Sciences - Jodhpur

Mahendra Kumar Garg

All India Institute of Medical Sciences - Jodhpur

\section{Sanjeev Misra}

All India Institute of Medical Sciences - Jodhpur

\section{Research Article}

Keywords: COVID-19, SARS-CoV-2, evacuation, quarantine, asymptomatic, R0, India

Posted Date: May 29th, 2020

DOI: https://doi.org/10.21203/rs.3.rs-31529/v1

License: (1) (1) This work is licensed under a Creative Commons Attribution 4.0 International License.

Read Full License 


\section{Abstract \\ Background}

During COVID-19 pandemic, Indian nationals were evacuated from foreign countries to various quarantine facilities in India. Individuals arriving from Iran on 14 March 2020 were quarantined at Jaisalmer, Rajasthan. All individuals were tested for SARS-CoV-2 infection after completing 14 days of quarantine. Those testing positive were isolated at AllMS Jodhpur, India. We attempt to describe the transmission dynamics of SARS-CoV2 in this cohort.

\section{Methods}

Basic SEIR compartmental model was developed using daily stepwise approach in Microsoft Excel. Advanced model using standard differential equations in Python software version 3.6 was used to estimate $R O$ based on model fit to actual data.

\section{Results}

Forty-eight SARS-CoV-2 infections were found among the 474 evacuees. Out of them 44 (92\%) were asymptomatic. $R 0$ for the overall duration was found to be 2.29 ( $95 \% \mathrm{Cl} 1.84-2.78)$. Male gender and age greater than 60 years were associated with SARS-CoV-2 infection ( $R R=4.33,95 \% \mathrm{Cl} 2.07-9.05$ and 5.32, $95 \% \mathrm{Cl} 3.13-9.04$, respectively). Isolation of infected individuals and stricter quarantine of remaining individuals reduced the $R 0$ from 2.41 initially to 1.17 subsequently.

\section{Conclusion}

$R O$ value was found comparable to the earlier studies indicating similar transmission dynamics among quarantined individuals in India. Further, universal testing and prompt isolation of infected individuals was found effecting in interrupting the transmission of SARS-CoV-2. Role of asymptomatic individuals in transmission appears to be strong in the context of quarantine of evacuees.

\section{Introduction}

As on 22 May 2020, the COVID-19 pandemic has resulted in around five million cases globally and 0.32 million deaths [1]. It is imperative to understand the epidemiology and transmission dynamics of the disease, in order to guide the prevention strategy. Outbreaks in closed susceptible populations have provided an opportunity to understand the infection transmission of SARS-CoV-2 [2-7]. These included the Diamond Princess cruise ship harboured at Japan and skilled nursing facilities in the United States [2-7]. In India, decision was taken in March 2020 to evacuate Indian nationals stranded in foreign countries due to restriction on international travel. The evacuees had been screened for SARS-CoV-2 
infection by RT-PCR test prior to boarding the aircraft. Upon arrival, they were quarantined at facilities across India. The Indian Army facility at Jaisalmer, Rajasthan quarantined the evacuees arriving from Tehran, Iran on 14 March 2020.

The asymptomatic quarantined individuals at Jaisalmer were being prepared for release on 28 March 2020 , after completing 14 days of quarantine. However, upon request of the state government, all evacuees irrespective of symptoms were tested for SARS-CoV-2 infection with nasopharyngeal and oropharyngeal swabs by RT-PCR, from 28 March - 1 April 2020. Repeat testing of evacuees who had tested negative initially was conducted after 1 April 2020. Those who tested positive were admitted in isolation wards at All India Institute of Medical Sciences (AIIMS), Jodhpur, Rajasthan. They were further tested twice on days 14 and 15 of initial positive test result as per extant national guidelines [8]. They were discharged and allowed to travel to their home districts in India if both the test results were found negative.

\section{Methods}

We utilized the compartmental SEIR model, wherein S, E, I, R respectively denote the susceptible, the exposed, the infected and recovered or removed [2]. To begin with, all individuals were considered susceptible. Those testing positive at the quarantine facility were considered to move from 'susceptible' to 'infected' compartment on the day of sample collection and stayed there till the day of being isolated, which was usually a day after the declaration of test result. From date of isolation onwards they were considered 'removed'. The total number $\mathrm{N}=\mathrm{S}+\mathrm{E}+\mathrm{I}+\mathrm{R}$ remained fixed at each step. The input parameters of the model were first specified in time steps of 1 day starting from 14 March 2020. It was subsequently applied to a step-wise prediction model in Microsoft excel and standard differential equation model in Python software version 3.6 [2]. The differential equations were as follows:

$$
\begin{aligned}
& \frac{d S}{d t}=-\beta I \frac{S}{N} \\
& \frac{d E}{d t}=\beta I \frac{S}{N}-E \delta \\
& \frac{d I}{d t}=E \delta-\gamma I \\
& \frac{d R}{d t}=\gamma I
\end{aligned}
$$

Here, $\delta$ refers to reciprocal of incubation period equivalent to the rate of exposed turning infectious per day. Similarly, $y$ refers to rate of infectious individuals recovering per day (1/D). The relationship between $R O$ and duration of infectiousness (D) is expressed as per the following equation:

$$
\beta=\text { transmissibility } X \text { contact rate }=R 0 / D
$$

Where, $\beta$ referred to the transmissibility multiplied by contact rate or number of secondary cases generated by an infectious case per day. 


\section{Step-wise model in Microsoft Excel}

The objective of this basic model was to use backward extrapolation in the step-wise compartmental model to provide an estimate of initially infected individuals while considering known infection transmission parameters.

A mean $R 0$ value of 3.28 was taken from average of previous studies [9]. A duration of infectiousness of 10 days yielded the $\beta$ as 0.328 [10]. Median incubation period was taken as 5.5 days [10]. Thus, $\delta$ and $\gamma$ values were taken as $0.182 /$ day and $0.1 /$ day, respectively, for the basic step-wise model. This initial number of infected at day 1 (14 March 2020) was varied so as to achieve the observed number of SARSCov-2 positive individuals till all the evacuees were tested by 1 April 2020 (day 19).

\section{Differential equation model in Python v3.6}

The model was first specified in Python software with the standard differential equations of SEIR model [2]. Model was optimized to provide best fit using maximum likelihood approach with the input parameters so that the value of $R O$ could be estimated. Further, in order to visualize the effect of removal of positives and stricter avoidance of mixing on transmission, $R O$ value was assessed separately for the first wave when all individuals had been tested and subsequently when repeat testing was done. The prior values of $\delta, \gamma$ and $R O$ were same as in the basic model [10].

\section{Results}

Initially, 528 individuals were quarantined at Jaisalmer facility. Among them, 54 people who were accommodated separately and tested negative for SARS-CoV-2 after completing 14 days in quarantine and were sent home early. Therefore, only the remaining 474 individuals who were accommodated together were considered for studying the transmission of SARS-CoV-2. Of these 474 individuals, 254 were males (53.6\%). Around half of the evacuees belonged to the 15-29 years age group (Table 1).

Table 1

Age and gender distribution of the evacuees

\begin{tabular}{|lllllll|}
\hline Age category & \multicolumn{2}{l}{ Female } & \multicolumn{3}{l}{ Male } & \multicolumn{3}{l|}{ Total } \\
\cline { 2 - 7 } (in years) & $\mathbf{N}$ & $\%$ & $\mathbf{N}$ & $\%$ & $\mathbf{N}$ & $\%$ \\
\hline $0-14$ & 6 & 2.7 & 2 & 0.8 & 8 & 1.7 \\
\hline $15-29$ & 126 & 57.3 & 101 & 39.8 & 227 & 47.9 \\
\hline $30-44$ & 17 & 7.7 & 50 & 19.7 & 67 & 14.1 \\
\hline $45-59$ & 29 & 13.2 & 40 & 15.7 & 69 & 14.6 \\
\hline 60 plus & 42 & 19.1 & 61 & 24.0 & 103 & 21.7 \\
\hline Grand Total & 220 & 100.0 & 254 & 100.0 & 474 & 100.0 \\
\hline
\end{tabular}


Male gender and age 60 years or more were significantly associated with being infected with SARS-CoV-2 (Table 2).

Table 2

Association of age and gender with SARS-CoV-2 infection

\begin{tabular}{|lllll|}
\hline Characteristics & $\begin{array}{l}\text { SARS-CoV-2 positive } \\
\mathbf{n} / \text { total }(\%)\end{array}$ & $\begin{array}{l}\text { SARS-CoV-2 negative } \\
\mathbf{n} / \text { total }(\%)\end{array}$ & $\begin{array}{l}\text { Risk ratio } \\
(95 \% \mathrm{Cl})\end{array}$ & p-value \\
\hline Gender & & & & \\
\hline Male & $40 / 254(15.7)$ & $214 / 254(84.3)$ & 4.33 & $<0.0001$ \\
\hline Female & $8 / 220(3.6)$ & $212 / 220(96.4)$ & - & \\
\hline Age group & & & $5.07-9.05)$ & \\
\hline 60 years and more & $28 / 103(27.2)$ & $75 / 103(72.8)$ & 5.32 & \\
\hline
\end{tabular}

In the first wave of testing from 28 March 2020-1 April 2020, all the 474 evacuees were tested irrespective of symptoms. Thirty-five individuals were detected positive in the first wave. Subsequently, repeat testing among individuals who had tested negative earlier detected 13 new infections. Therefore, total 48 individuals were eventually found infected with SARS-CoV-2 (Fig. 1). Only four (8.3\%) were found to have fever. No other symptoms were reported. Mean stay in hospital isolation was $15.5 \pm 3.6$ days.

The step-wise model estimated that an initial size of 11 infected individuals was sufficient to result in 35 infected individuals by 1 April 2020, by the time first wave of testing was completed (Supplementary File 1).

In the differential equation model, the overall $R 0$ was estimated to be 2.29 ( $95 \% \mathrm{Cl} 1.84-2.78$ ). For the individuals detected positive in the first wave of testing, the $R 0$ value was 2.41 ( $95 \% \mathrm{Cl} 0.53-4.86$ ) which subsequently reduced to $1.17(95 \% \mathrm{Cl} 0.87-1.24)$. The best fit curve of infected individuals overall and separately in the first and second waves was also obtained (Fig. 2, Fig. 3). Further details of the Python 3.6 code used for analysis are provided in Supplementary file 2 .

\section{Discussion}

Male gender and older age were found to be significantly associated with SARS-CoV-2 infection. Although men and older individuals had been found to be at risk of severe COVID-19 [11, 12], it remains unclear whether they are also at higher risk of SARS-CoV-2 infection. Assortative mixing of men of older age 
group in the quarantine facility could have played a role in this association, rather than male gender and older age being true risk factors of SARS-CoV-2 infection.

The overall $R 0$ value of 2.29 found in our study was comparable to previous estimates reported mainly from China in the early phase of the COVID-19 pandemic [9]. The reduction in $R 0$ estimate once infected individuals were isolated and stricter physical separation was ensured, was similar to the finding from the Diamond Princess cruise ship $[2,13]$. During the early phase of the pandemic, embarkation of passengers on ships at specific ports had enabled ascertainment of index case with reasonable accuracy [5]. On the other hand, it becomes difficult to establish the index case for evacuations when all the individuals usually start travelling together from the origin country.

The evacuees were brought to India after being screened for SARS-CoV-2 infection. However, the evacuation situation provides a unique challenge wherein large number of susceptible individuals are brought within confined spaces such as during transit to the airport, waiting areas at the airport and to the quarantine facility. Therefore, the stage of the outbreak in which evacuation is being carried out appears to be important. If evacuation is done when transmission is well established in the source country, there would be a higher risk of infecting more susceptible individuals during the process. Consequently, stricter safeguards might be required. Therefore, decision to evacuate must carefully take in account these epidemiological factors. Further, the rationale of universal screening prior to evacuation mainly depends on the test having perfect accuracy and having ability to detect all individuals shedding the virus.

Although a negative RT-PCR test for SARS-CoV-2 infection was considered mandatory for evacuation, the step-wise model estimated that around $2.3 \%$ of the evacuees $(11 / 474)$ could have been infected prior to arrival in India. The RT-PCR test used for screening has been found to have 2-29\% false negatives [14]. Further, in the event of a negative test, the post-test probability of actually being negative depends on the pre-test probability [15]. The pre-test probability of a test in turn corresponds to the prevalence of infection in the source population [16]. Given that evacuation happened from Iran when COVID-19 outbreak was well established, it would be reasonable to assume an infection prevalence of at least $5 \%$ the source population, similar to sero-prevalence reported elsewhere [17]. Thus, $1.6 \%$ evacuees could have been false-negative [15]. Due to the considerable proportion of asymptomatic SARS-CoV-2 infections, mandatory screening of all individuals irrespective of symptoms upon completion of quarantine appeared to be the right decision.

We found that another 13 quarantined individuals who had tested negative in the first wave of testing turned positive in repeat testing. It is likely that they were incubating when the testing was done initially for all individuals. Therefore, once transmission has started, even testing all evacuees at a single point of time might not prove sufficient. Hence, it becomes all the more important to maintain adequate physical separation, ensure hand hygiene and strict avoidance of mixing to prevent the initial flare-up of transmission during quarantine. 
Further, our observation of more than $90 \%$ asymptomatic infection among evacuees matches the higher bound of $18-88 \%$ asymptomatic SARS-CoV-2 infection found in other studies $[3,7,18,19]$. It also further supports the role of asymptomatic or mildly symptomatic carriers in transmitting the SARS-CoV-2 infection [20]. Existing modelling techniques are well suited to the input of daily incidence data which is easy to obtain for symptomatic cases. Our study had the limitation that daily testing and incidence data could not be used in the situation of mass testing of individuals irrespective of symptoms. Also, our population size was smaller and we had lesser number of data points. Therefore, we had comparatively larger error estimates for the time-dependent $R 0$ values derived.

\section{Conclusion}

$R 0$ estimate for SARS-CoV-2 infection found in the Jaisalmer quarantine camp indicates similar transmission pattern as in China during the early phase of the pandemic in January 2020. Testing all individuals irrespective of symptoms and prompt isolation of infected individuals effectively interrupted the transmission of SARS-CoV-2 in the quarantine facility. Therefore, this strategy is recommended for routine implementation in all quarantine situations following overseas travel, so that transmission could be prevented in the general population. This becomes especially important with the gradual resumption of international travel. Role of asymptomatic individuals in transmission appears to be strong in the context of quarantine of evacuees. Therefore, universal screening in closed populations and contact screening generally appear to be more effective prevention strategies as compared to symptomatic screening.

\section{Abbreviations}

AllMS Jodhpur

All India Institute of Medical Sciences, Jodhpur, India

COVID-19

Corona Virus Disease 2019

$R 0$

basic reproduction number

RT-PCR

Reverse Transcriptase Polymerase Chain Reaction

SARS-CoV-2

Severe Acute Respiratory Syndrome Corona Virus 2

SEIR

Susceptible, Exposed, Infected and Recovered (or removed)

\section{Declarations}

Ethics approval and consent to participate - Since the study was based retrospective analysis based on routinely collected data, it was exempted from the requirement for institutional ethics committee review. 
Consent for participation - Not applicable.

Availability of data and materials - All data are presented in the article and as supplementary filed.

Competing interests - The authors declare that there are no competing interests for

publication of this article. The views expressed in this article are those of the authors

alone and do not necessarily represent the views of their respective organizations.

Authors' contributions - MKG conceived the idea of the study. SS extracted the data and developed the step-wise model. RK developed the advanced model in Python and did the analysis. SS wrote the draft manuscript draft with further inputs from MKG, PB, NK and SM. PB coordinated the data collection and VLN coordinated the testing of samples. SM provided overall supervision of the testing, isolation, clinical care and research related to COVID-19. All authors approved the final manuscript.

Funding - The authors declare that no funding was received from any source for the study and preparation of this article.

Acknowledgements - We acknowledge the help of personnel and staff operating the Jaisalmer quarantine centre and the staff at AlIMS Jodhpur involved in testing and clinical care of evacuees.

\section{References}

1. WHO. Situation Update no. 122 for COVID-19-21. May 2020. World Health Organization, Geneva; 2020. Available at: https://www.who.int/docs/default-source/coronaviruse/situationreports/20200521-covid-19-sitrep-122.pdf?sfvrsn=24f20e05_2 (accessed 22 May 2020).

2. Rocklöv J, Sjödin H, Wilder-Smith A. COVID-19 outbreak on the Diamond Princess cruise ship: estimating the epidemic potential and effectiveness of public health countermeasures. J Travel Med 2020; 27 (3). https://doi.org/10.1093/jtm/taaa030.

3. Arons MM, Hatfield KM, Reddy SC, Kimball A, James A, Jacobs JR, et al. Presymptomatic SARS-CoV2 Infections and Transmission in a Skilled Nursing Facility. N Engl J Med. 2020. https://doi.org/10.1056/NEJMoa2008457.

4. Leung WS, Chan JMC, Chik TSH, Lau DPL, Choi CYC, Lau AWT, et al. Presumed COVID-19 Index Case on Diamond Princess cruise ship and Evacuees to Hong Kong. J Travel Med 13 May 2020. https://doi.org/10.1093/jtm/taaa073.

5. Russell TW, Hellewell J, Jarvis Cl, van Zandvoort K, Abbott S, Ratnayake R, et al. Estimating the infection and case fatality ratio for coronavirus disease (COVID-19) using age-adjusted data from the outbreak on the Diamond Princess cruise ship, February 2020. Euro Surveill. 2020;25(12):pii = 2000256. https://doi.org/10.2807/1560-7917.ES.2020.25.12.2000256. 
6. Zhang S, Diao M, Yu W, Pei L, Lin Z, Chen D. Estimation of the reproductive number of novel coronavirus (COVID-19) and the probable outbreak size on the Diamond Princess cruise ship: A datadriven analysis. Int $\mathrm{J}$ Infect Dis. 2020;93:201-4.

7. Mizumoto K, Kagaya K, Zarebski A, Chowell G. Estimating the asymptomatic proportion of coronavirus disease 2019 (COVID-19) cases on board the Diamond Princess cruise ship, Yokohama, Japan, 2020. Euro Surveill. 2020;25(10):pii = 2000180. https://doi.org/10.2807/15607917.ES.2020.25.10.2000180.

8. ICMR. Revised strategy of COVID-19 testing in India - version 3 (dated 20 March 2020). Indian Council of Medical Research, New Delhi; 2020. Available at: https://main.icmr.nic.in/sites/default/files/upload_documents/2020-03-20_covid19_test_v3.pdf (accessed 18 May 2020).

9. Liu Y, Gayle AA, Wilder-Smith A, Rocklöv J. The reproductive number of COVID-19 is higher compared to SARS coronavirus. J Travel Med 2020: 27(2). https://doi.org/10.1093/jtm/taaa021.

10. Li Q, Guan X, Wu P, Wang X, Zhou L, Tong Y, et al. Early Transmission Dynamics in Wuhan, China, of Novel Coronavirus-Infected Pneumonia. N Engl J Med. 2020;382:1199-207.

11. Meng Y, Wu P, Lu W, Liu K, Ma K, Huang L, et al. Sex-specific clinical characteristics and prognosis of coronavirus disease-19 infection in Wuhan, China: A retrospective study of 168 severe patients. PLOS Pathogens. 2020;16:e1008520.

12. https:// Zhang J-J, Dong X, Cao Y-Y, Yuan Y-D, Yang Y-B, Yan Y-Q, et al. Clinical characteristics of 140 patients infected with SARS-CoV-2 in Wuhan, China. Allergy 2020. https://doi: 10.1111/all.14238.

13. Liu F, Li X, Zhu G. Using the contact network model and Metropolis-Hastings sampling to reconstruct the COVID-19 spread on the “Diamond Princess. Sci Bull (Beijing). 2020. doi:10.1016/j.scib.2020.04.043.

14. Arevalo-Rodriguez I, Buitrago-Garcia D, Simancas-Racines D, Zambrano-Achig P, Campo R del, Ciapponi A, et al. False-negative results of initial RT-PCR assays for COVID-19: A systematic review. medRxiv 2020;2020.04.16.20066787.

15. Watson J, Whiting PF, Brush JE. Interpreting a covid-19 test result. BMJ. 2020;369:m1808.

16. Grimes DA, Schulz KF. Uses and abuses of screening tests. Lancet. 2002;359:881-4.

17. Treibel TA, Manisty C, Burton M, McKnight Á, Lambourne J, Augusto JB, et al. COVID-19: PCR screening of asymptomatic health-care workers at London hospital. Lancet. 2020;395:1608-10. https://doi.org/10.1016/S0140-6736(20)31100-4.

18. Sutton D, Fuchs K, D'Alton M, Goffman D. Universal Screening for SARS-CoV-2 in Women Admitted for Delivery. N Engl J Med. 2020. https://doi.org/10.1056/NEJMc2009316.

19. Gudbjartsson DF, Helgason A, Jonsson H, Magnusson OT, Melsted P, Norddahl GL, et al. Spread of SARS-CoV-2 in the Icelandic Population. N Engl J Med. 2020. https://doi.org/10.1056/NEJMoa2006100. 
20. Bai Y, Yao L, Wei T, Tian F, Jin D-Y, Chen L, et al. Presumed Asymptomatic Carrier Transmission of COVID-19. JAMA. 2020;323:1406-7.

\section{Supplementary Materials}

Supplementary file 1: Basic step-wise SEIR model for SARS-CoV-2 infection among evacuees quarantined at Jaisalmer

Supplementary file 2: Code and results of SEIR model in Python version 3.6

\section{Figures}

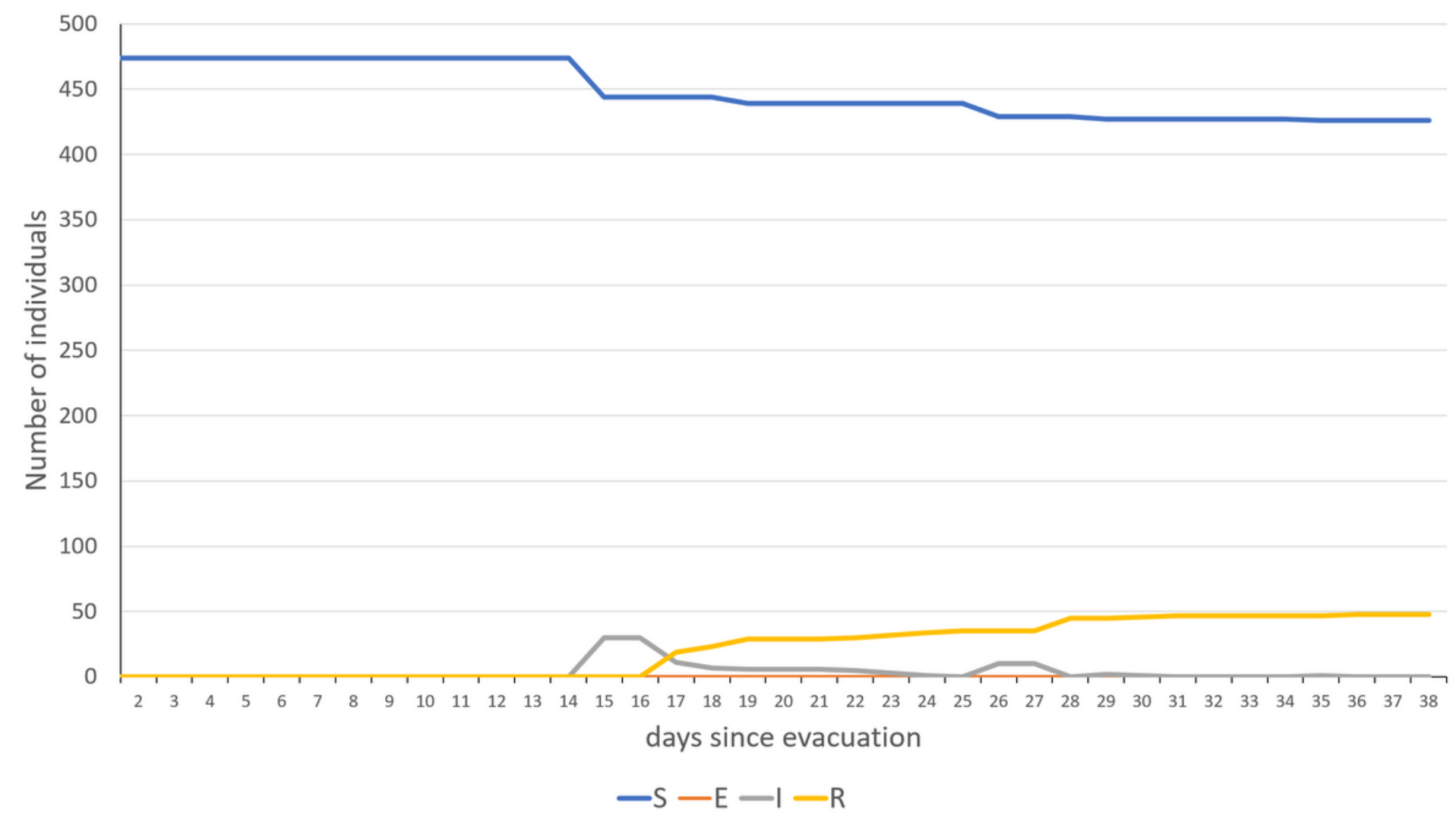

\section{Figure 1}

Progression of SARS-CoV-2 infection (SEIR model) for the 474 individuals quarantined at Jaisalmer facility 


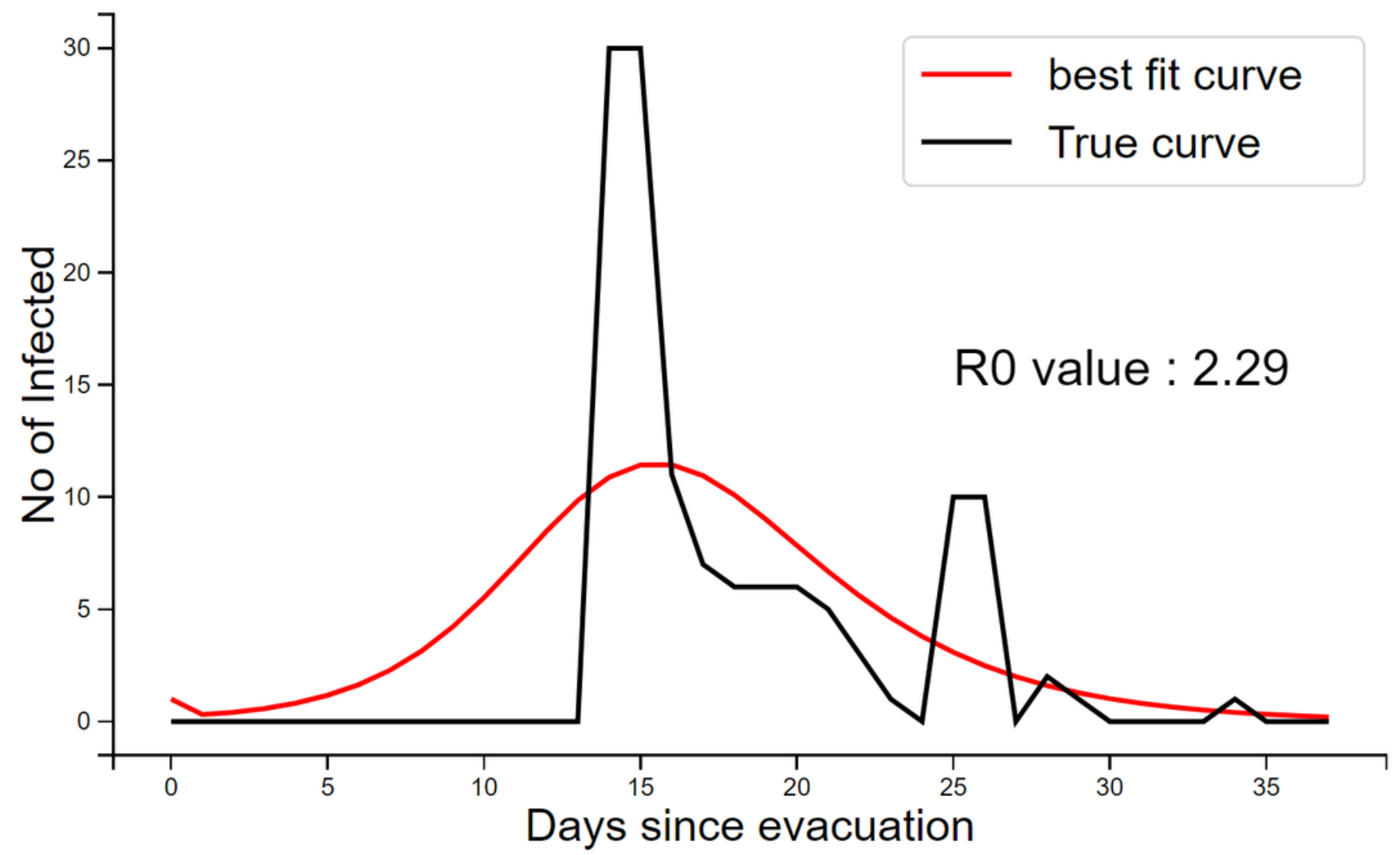

Figure 2

Number of infected individuals - observed and fitted to gamma distribution and overall R0 value
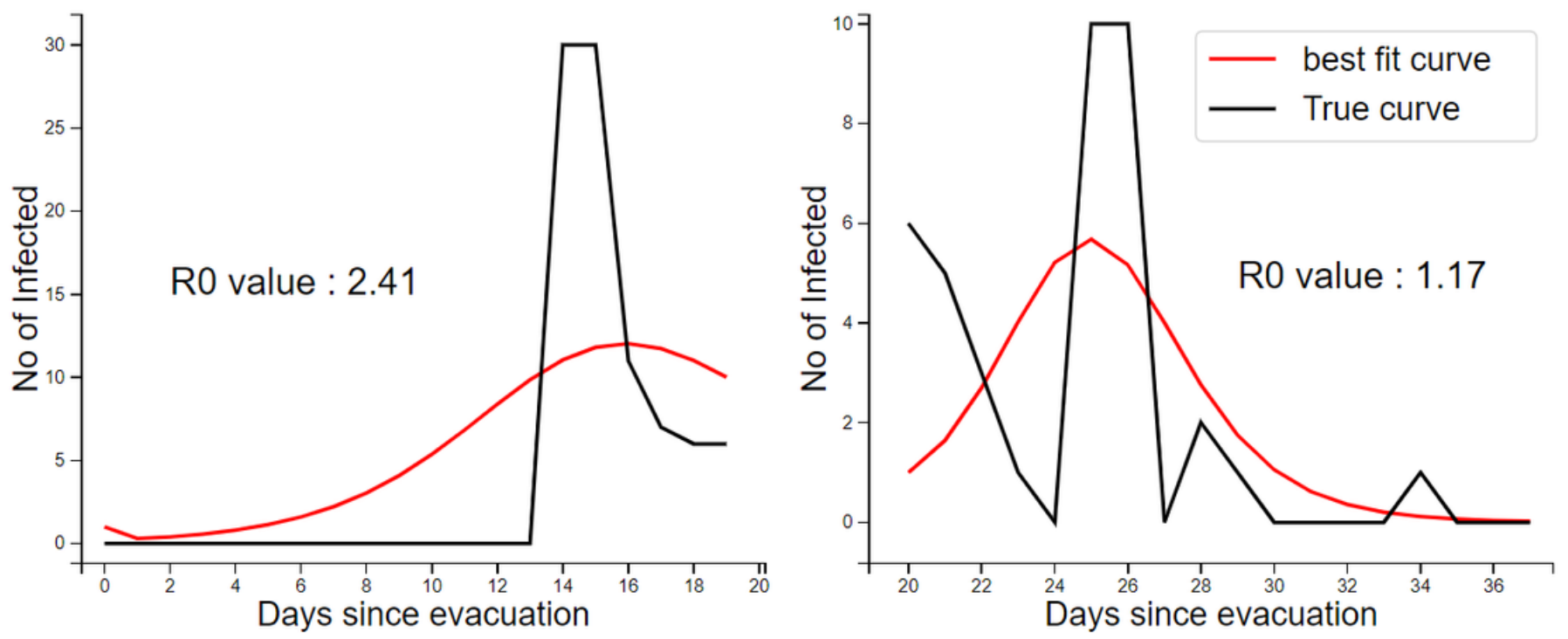

Figure 3

Number of infected individuals - observed and fitted to gamma distribution and R0 value in first wave of testing and later 


\section{Supplementary Files}

This is a list of supplementary files associated with this preprint. Click to download.

- Supplementaryfile1.xlsx

- Supplementaryfile2.html 